\title{
Spin Transfer Torque Magnetization Switching in Ferromagnetic Nanopillars with Orange Peel Coupling
}

\author{
D. Aravinthan ${ }^{1, *}$, P. Sabareesan ${ }^{2}$, M. Daniel ${ }^{3}$ \\ ${ }^{1}$ Centre for Nonlinear Dynamics, School of Physics, Bharathidasan University, Tiruchirappalli - 620024 \\ ${ }^{2}$ Centre for Nonlinear Science and Engineering, School of Electrical and Electronics Engineering, SASTRA \\ University, Thanjavur - 613401 \\ ${ }^{3}$ SNS Institutions, Coimbatore - 641 049, India
}

\begin{abstract}
We have studied the effect of orange peel coupling on spin transfer torque magnetization switching in different nanopillar devices. The magnetization switching dynamics of the free layer of the nanopillar device is studied by solving the dynamical equation governed by a Landau-Lifshitz-Gilbert-Slonczewski (LLGS) equation. The switching time is calculated for $\mathrm{Fe}$, Co, Ni and NiFe (Py) materials both in the presence and the absence of orange peel coupling. Presence of orange peel coupling between the ferromagnetic layers reduces the switching time for all the materials. Fe material shows the highest switching time value whereas Py material shows the lowest switching time.
\end{abstract}

Keywords: magnetization switching, nanopillar, orange peel coupling, spin transfer torque, spintronics,

\section{Introduction}

Recently spin transfer torque (STT) magnetization switching in magnetic nanopillar devices have found applications in microwave frequency generators, high density read heads and nonvolatile magnetic random access memories [1]. STT phenomenon was first suggested by Slonczewski [2] and Berger [3] independently. Trilayer nanopillar device consisting of two ferromagnetic layers (pinned layer and free layer) separated by a nonmagnetic spacer layer is the basic structure used in the memory devices. Reducing the switching time of the free layer magnetization is one of the important issue to develop potential applications. Growing nanopillar for memory applications without roughness is a difficult task. Hence, the resultant nanopillar have certain interface roughness and they give rise to two different coupling mechanisms [4]. First one is Neel coupling or orange peel coupling that arises in situations where the spacer layer has a uniform thickness with a correlated roughness [5]. Second one is the biquadratic coupling which occurs when the roughness of the pinned and free layer are uncorrelated [6]. We have recently studied the impact of both orange peel coupling [7] and biquadratic coupling [8] on STT switching in $\mathrm{Co} / \mathrm{Cu} / \mathrm{NiFe}$ nanopillar. Motivated by this, in this paper, we study the effect of orange peel coupling in $\mathrm{Co} / \mathrm{Cu} / \mathrm{X}$ (where $\mathrm{X}$ represents $\mathrm{Fe}, \mathrm{Co}, \mathrm{Ni}$ and $\mathrm{NiFe}$ (Py)) nanopillars which have been used for memory devices. This is carried out by solving the magnetization switching dynamics of the free layer governed by the Landau-Lifshitz-Gilbert-Slonczewski (LLGS) equation. The paper is organized as follows. Description about the geometry of the trilayer nanopillar and construction of the dynamical equation are presented in section II. Numerical simulation results and their discussion are given in section III. Finally concluding remarks are made in section IV.

\section{Model and Dynamical Equation}

The trilayer nanopillar considered for our study consists of two ferromagnetic layers (FM1, FM2) separated by a nonmagnetic (NM) spacer layer. A schematic sketch of the trilayer nanopillar device is shown in Fig. 1. First ferromagnetic layer (FM1) is made up of high coercivity material cobalt (Co) in order to filter the electron's spin to produce a spin polarized current. The thickness of the FM1 layer is $4 \mathrm{~nm}$ and its magnetization $\left(\mathbf{m}_{\mathbf{p}}\right)$ is pinned and parallel to the plane of the nanopillar. Magnetization of the second ferromagnetic layer (FM2) is free to move, when the current is passed through them and hence it is called as a "free layer". Magnetization of the free layer has in-plane magneto-crystalline anisotropy and thickness of the free layer is 4 $\mathrm{nm}$. The middle spacer layer is made up of nonmagnetic metal copper $(\mathrm{Cu})$ and its thickness $(2 \mathrm{~nm})$ is small 


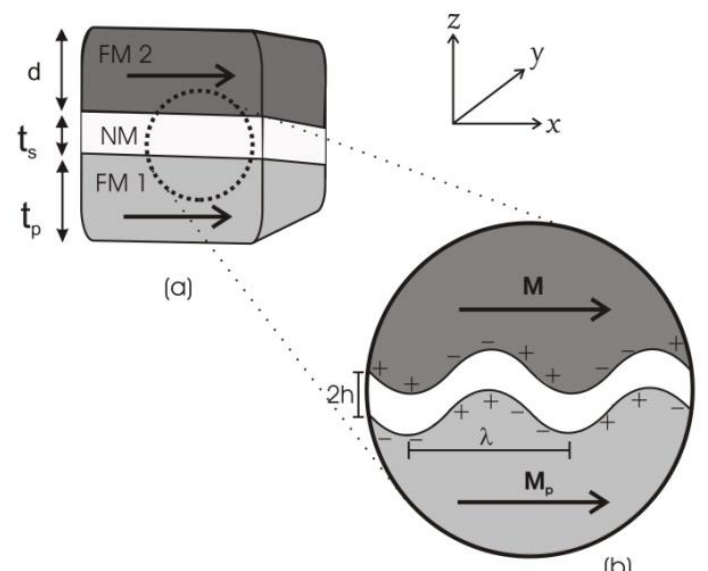

Figure 1: (a). A sketch of the trilayer nanopillar device. FM1 is the pinned layer and FM2 is the free layer. NM represents the nonmagnetic spacer layer. $\mathrm{d}$, ts and tp are the thickness of the free layer, spacer layer and pinned layer respectively. (b). In the zoomed view, we can see the nonmagnetic spacer layer interface has the correlated waviness with wavelength $\lambda$ and amplitude $\mathrm{h}$. $\mathbf{M}$ and $\mathbf{M p}$ represent the magnetization of the free layer and pinned layer respectively. When the magnetization of two ferromagnetic layers are parallel, magnetostatic charges $(-,+)$ of opposite sign $(+,-)$ appear symmetrically on opposing interfaces.

Enough to transfer the polarized current from the pinned layer to free layer. The nonmagnetic spacer has the correlated waviness interface with wavelength $\lambda=40 \times 10^{-9} \mathrm{~m}$ and amplitude $\mathrm{h}=0.8 \times 10^{-9} \mathrm{~m}$. Initially both pinned and free layer magnetizations are aligned parallel to each other and hence magnetic charges of opposite sign appear symmetrically on the opposing interfaces. The dipole interaction between these opposing charges gives rise to a orange peel coupling referred so because of the dimpled texture of an orange. Current is applied normal to the of device plane (z-direction) and it becomes spin polarized while passing through the pinned layer. The spin polarized current entered into the free layer via spacer layer produces a STT due to exchange interaction between the spins of conduction electrons and local magnetization. This STT will switch the magnetization of the free layer when the applied current is above the threshold value. The magnetization switching dynamics of the free layer is governed by LLGS equation and it can be written in dimensionless form as [7],

$$
\begin{aligned}
& \frac{d m}{d \tau}=-\left(m \times h_{e f f}\right)-\alpha\left[m \times\left(m \times h_{\text {eff }}\right)\right]+a_{j}\left[m \times\left(m \times m_{1}\right.\right. \\
& m^{2}=m^{x 2}+m^{y 2}+n=1 .
\end{aligned}
$$

Here, is the Gilbert damping parameter, $\mathbf{m}=\quad$ is the dimensionless magnetization vector of the free layer,

$\boldsymbol{m}_{p}=$ is the dimensionless unit magnetization of the pinned layer, is saturation magnetization of the free layer and its value is different for different materials and $a_{j}=\frac{P_{j}}{H_{0} e_{i}}$ is the spin transfer torque coefficient. Here, $\mathrm{p}$ is the polarization factor, $\mathrm{J}$ is the current density applied, is the permeability of the free space, e is the electron's charge, $\mathrm{d}$ is the thickness of the free layer. $\tau=\gamma l$ is the dimensionless time, where is gyromagnetic ratio. $h$ is the effective magnetic field acting on the free layer and it can be written as,

$$
h=h_{\text {ma }}+h_{\text {shape }}+h_{\text {ext }}+h \text {. }
$$

Magnetization of the free layer taken for our study is aligned along its easy axis (x-axis) and hence magnetocrystalline anisotropy acts along $\mathrm{x}$-axis. The field due to magneto-crystalline anisotropy can be written as, $\boldsymbol{h}_{\text {ma }}=h_{a} \boldsymbol{m}^{x}$ where is the unit vector along $\mathrm{x}$-direction and $h_{a}=\frac{\tilde{z}}{\mathfrak{G}}$. Here, $\mathrm{k}_{\mathrm{a}}$ is the magnetocrystalline anisotropy coefficient. $\quad \boldsymbol{h}_{\text {sh }}$ is the field term corresponding to the shape anisotropy. In our case, the free layer lies in the xy-plane and hence the value of demagnetization factors becomes, $\mathrm{N}_{\mathrm{x}}=\mathrm{N}_{\mathrm{y}}=0$, and $\mathrm{N}_{\mathrm{z}}=1$. Therefore, shape anisotropy field can be written as, $\quad \boldsymbol{h}_{\mathrm{ghape}}=-\mathrm{N}_{z} m^{z}$ where is the unit vector along $\mathrm{z}$ direction. The free and pinned layer magnetizations are initially aligned along the easy axis and perpendicular to the roughness. Hence the orange peel coupling occur perpendicular to the easy axis and the field strength of the coupling can be written as $\quad h_{o p c}=h_{n} m$. Here, $\quad h_{n}=\frac{\pi^{2} \pi^{2}}{\sqrt{2} \pi d} \exp \left(\frac{-2 \sqrt{2} \pi}{\mathrm{A}}\right.$ is the 
magnitude of the coupling field strength called as Néel field. When an external magnetic field $h_{e}$ is applied perpendicular to the easy axis (along y-direction), then $\boldsymbol{h}_{e x t}=h_{e} \boldsymbol{e}^{\boldsymbol{y}}$. Hence, The total effective field acting on the free layer can be written as,

$$
\boldsymbol{h}_{\text {eff }}=h_{a} m^{x} e^{x}+\left(h_{e}+h_{n} m^{y}\right) e^{y}-N_{z} m \text {. }
$$

By substituting the effective field found in Eq. (3) into Eq. (1), we obtain the dynamical equation for our study. Numerical simulation results of the LLGS equation and discussion of the results are presented in the forthcoming section.

\section{Results and Discussion}

The magnetization switching dynamics of the free layer is governed by the LLGS equation (Eq. 1) and it is studied for $\mathrm{Fe}, \mathrm{Co}, \mathrm{Ni}$ and Py materials by numerically integrating the LLGS equation using Runge-Kutta fourth order (RK4) procedure. In order to find the effect of orange peel coupling on switching time, the LLGS equation is computed both in the presence and in the absence of orange peel coupling case separately for each material. The values of various material parameters used for numerical simulations are given in Table 1 [9]. The value of the Gilbert damping parameter and polarization factor is chosen as $\alpha=0.001$ and $\mathrm{p}=0.4$ respectively to suppress the ringing effect.

Table 1: Various material parameters used in the numerical simulations

\begin{tabular}{|l|c|c|c|c|}
\hline \multicolumn{1}{|c|}{ Parameters } & $\mathrm{Fe}$ & $\mathrm{Co}$ & $\mathrm{Ni}$ & $\mathrm{NiFe}$ \\
\hline $\begin{array}{l}\text { Magneto-crystalline anisotropy } \mathrm{K}_{\mathrm{c}}(\times \\
\left.10^{3} \mathrm{Jm}^{-3}\right)\end{array}$ & 48 & 500 & -5.7 & 2 \\
\hline $\begin{array}{l}\text { Saturation magnetization } \\
\mathrm{M}_{\mathrm{s}}\left(\times 10^{6} \mathrm{Am}^{-1}\right)\end{array}$ & 1.718 & 1.449 & 0.493 & 0.795 \\
\hline
\end{tabular}

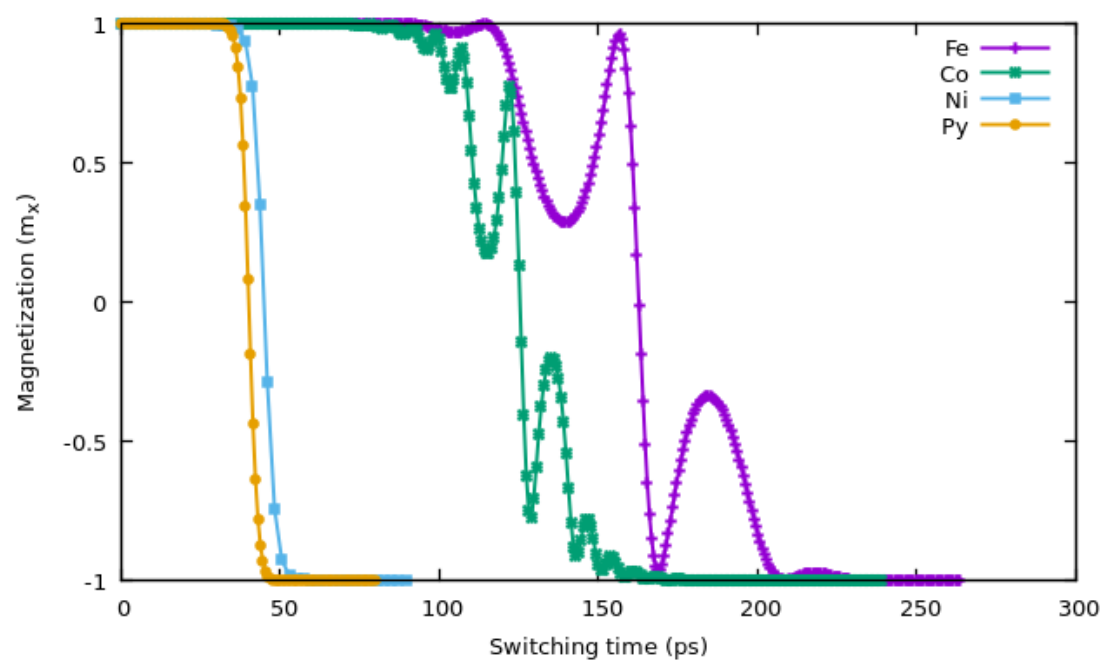

Figure 2: A plot of free layer magnetization versus switching time for the $\mathrm{Co} / \mathrm{Cu} / \mathrm{X}$ nanopillar in the absence of the orange peel coupling for an applied current density of $\mathrm{J}=4 \times 10^{12} \mathrm{Am}^{-2}$.

Table 2: Switching time in the absence and in the presence of orange peel coupling for an applied current

\begin{tabular}{|l|c|c|c|c|}
\hline \multicolumn{1}{|c|}{ Material } & $\mathrm{Fe}$ & $\mathrm{Co}$ & $\mathrm{Ni}$ & $\mathrm{NiFe}$ \\
\hline $\begin{array}{l}\text { Switching time in the absence of } \\
\text { orange peel coupling (in ps) }\end{array}$ & 257 & 204 & 76 & 68 \\
\hline $\begin{array}{l}\text { Switching time in the presence of } \\
\text { orange peel coupling (in ps) }\end{array}$ & 205 & 184 & 58 & 49 \\
\hline
\end{tabular}




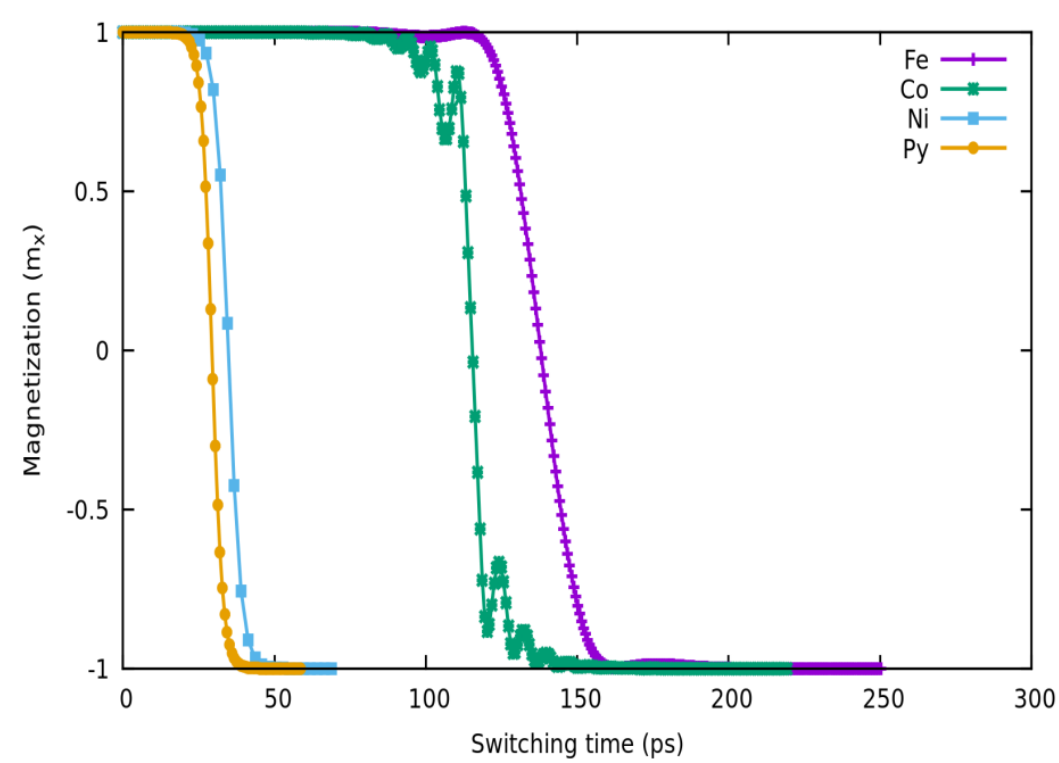

Figure 2: A plot of free layer magnetization versus switching time for the $\mathrm{Co} / \mathrm{Cu} / \mathrm{X}$ nanopillar in the presence of the orange peel coupling for an applied current density of $\mathrm{J}=4 \times 10^{12} \mathrm{Am}^{-2}$.

For the $\mathrm{Co} / \mathrm{Cu} / \mathrm{X}$ nanopillar where $\mathrm{X}$ represents the material of the free layer $(\mathrm{X}=\mathrm{Fe}, \mathrm{Co}, \mathrm{Ni}$ and $\mathrm{Py})$, the free layer magnetization is plotted against the switching time in the absence of orange peel coupling for the applied current density of $\mathrm{J}=4 \times 10^{12} \mathrm{Am}^{-2}$ from the numerical simulation which is shown in Fig. 2 . The free layer magnetization against the switching time in the presence of orange peel coupling is plotted in Fig. 3. The switching time for each material both in the absence and in the presence of orange peel coupling is calculated from numerical study and its values are given in Table 2. For all the materials, the switching time reduces when there is a orange peel coupling between the ferromagnetic layers. The reason is that, in the presence of orange peel coupling, additional magnetic field is generated due to orange peel coupling which combined with STT moves the magnetization of the free layer from in-plane to out of plane very fast. This generates a demagnetizing field due to a shape anisotropy which pulls the magnetization to the in-plane (in the switched state) from the out of plane. The switching time is higher for $\mathrm{Fe}$, because saturation magnetization value of $\mathrm{Fe}$ is high and hence time taken to initiate the switching is also high. Switching curves of both $\mathrm{Fe}$ and Co have some oscillations due to the ringing effect, which arises because of their high saturation magnetization value. The lowest switching time for both in the presence and in the absence of orange peel coupling is obtained for NiFe (Py) material. The reason is that value of the magneto-crystalline anisotropy and saturation magnetization is low for Py compared to the other materials. Hence, the switching time can be reduced by fabricating the free layer of the nanopillar with very low magneto-crystalline anisotropy and saturation magnetization and with orange peel coupling.

\section{Conclusion}

In this paper, we have studied the spin transfer torque magnetization switching and the effect of orange peel coupling on it for different nanopillar devices by numerically solving the LLGS equation which represents the magnetization switching dynamics of the free layer of the nanopillar devices. The switching time is calculated for $\mathrm{Fe}, \mathrm{Co}, \mathrm{Ni}$ and Py materials in the presence and the absence of orange peel coupling. Presence of orange peel coupling between the pinned layer and free layer reduces the switching time for all the materials. Fe shows the highest switching time value whereas Py shows the lowest switching time. Magnetization switching speed of the free layer can be enhanced by fabricating very low magneto-crystalline anisotropy and saturation magnetization material having orange peel coupling as the free layer of the nanopillar device.

\section{Acknowledgements}

D. A acknowledges Department of Science and Technology (DST), Govt. of India for the award of DSTINSPIRE Fellowship. P. S acknowledges DST for the award of SERB - Young Scientist project (SB/FTP/PS061/2013). 


\section{References}

[1]. C. Chappert, A. Fert, and F. N. Van Dau, The emergence of spin electronics in data storage, Nat. Mater. 6, 2007, 813-823.

[2]. J. C. Slonczewski, Current-driven excitation of magnetic multilayers, J. Magn. Magn. Mater., 159, 1996, L1-L7.

[3]. L. Berger, Emission of spin waves by a magnetic multilayer traversed by a current, Phys. Rev. B, 54, 9353-9358 (1996).

[5]. M. Stiles, Interlaer exchange coupling, in J. Bland and B. Heinrich (Ed.), Ultrathin Magnetic Structures III, (Springer Berlin Heidelberg, 2005) 99-142.

[6]. L. Néel, C. R. Acad. Sci. 255, 1676-1681 (1962).

[7]. S. Demokritov, E. Tsymbal, P. Grünberg, W. Zinn, and I. K. Schuller, Magnetic-dipole mechanism for biquadratic interlayer coupling, Phys. Rev. B 49, 1994, 720-723.

[8]. D. Aravinthan, P. Sabareesan, and M. Daniel, Current induced magnetization switching in $\mathrm{Co} / \mathrm{Cu} / \mathrm{Ni}-\mathrm{Fe}$ nanopillar with orange peel coupling, AIP Advances 5, 2015, 077166.

[9]. D. Aravinthan, P. Sabareesan, and M. Daniel, Effect of biquadratic coupling on current induced magnetization switching in $\mathrm{Co} / \mathrm{Cu} / \mathrm{NiFe}$ nanopillar, AIP Conf. Proc. 1731, 2016, 130032.

[10]. P. Sabareesan, and M. Daniel, Analytical study of fast precessional switching of magnetization in ferromagnetic nanofilms, J. Phys.: Condens. Matter 23, 2011, 046004. 